\title{
Recent results on CP and CPT tests at KLOE
}

\author{
A. De Santis* on behalf of KLOE/KLOE-2 collaborations \\ Dipartimento di Fisica, Università di Roma 'La Sapienza' \\ and I.N.F.N. Sezione di Roma \\ P.le A. Moro, 2 \\ I-00185 Rome, Italy \\ E-mail: antonio.desantiseromal.infn.it
}

The neutral kaon system offers a unique possibility to perform fundamental tests of fundamental symmetry such $C P$ and $C P T$ invariance. In this contribution two KLOE measurements are shown: the upper limit on the branching fraction for $K_{S} \rightarrow 3 \pi^{0}$ and the $C P T$ symmetry test in the Standard Model Extension framework in association with Lorentz Invariance breaking. Preliminary results are reported for both measurements: $B R\left(K_{S} \rightarrow 3 \pi^{0}\right)<2.9 \times 10^{-8} @ 90 \%$ C.L., $\Delta a_{X}=(-6.3 \pm$ $6.0) \times 10^{-18} \mathrm{GeV}, \Delta a_{Y}=(2.8 \pm 5.9) \times 10^{-18} \mathrm{GeV}, \Delta a_{Z}=(2.4 \pm 9.7) \times 10^{-18} \mathrm{GeV}$. The results on SME parameters $\left(\Delta a_{\mu}\right)$ are based on half of the total KLOE statistics. In this contribution a novel method for this analysis will be also discussed. A brief description of the future plans for the KLOE-2 project will be given.

The XIth International Conference on Heavy Quarks and Leptons,

June 11-15, 2012

Prague, Czech Republic

${ }^{*}$ Speaker. 


\section{The KLOE experiment}

The KLOE experiment operates at DAФNE, the Frascati $\phi$-factory. DA $\Phi$ NE is an $e^{+} e^{-}$ collider running at a center of mass energy of $\sim 1020 \mathrm{MeV}$, the mass of the $\phi$ meson. Positron and electron beams collide at an angle of $\pi-25 \mathrm{mrad}$, producing $\phi$ mesons with small momentum in the orbit plane $\left(p_{x}(\phi) \sim-15 \mathrm{MeV}\right)$.

The KLOE detector consists of a large cylindrical drift chamber (DC) surrounded by a leadscintillating fiber electromagnetic calorimeter (EMC). A super-conducting coil around the EMC provides a $0.52 \mathrm{~T}$ axial field.

The DC[1] is $4 \mathrm{~m}$ in diameter and $3.3 \mathrm{~m}$ long and has 12,582 all-stereo tungsten sense wires. The chamber shell is made of carbon fiber-epoxy composite and the gas used is a $90 \%$ helium, $10 \%$ isobutane mixture. These features maximize transparency to photons and reduce $K_{L} \rightarrow K_{S}$ regeneration and multiple scattering. The position resolutions for single hits are $\sigma_{r, \phi} \sim 150 \mu \mathrm{m}$ and $\sigma_{z} \sim 2 \mathrm{~mm}$ almost omogeneus in the sensible area. Those performancies results in a decay vertex position resolution ranging between 3-6 $\mathrm{mm}$ in the entire KLOE internal volume. The momentum resolution is $\sigma\left(p_{\perp}\right) / p_{\perp} \sim 0.4 \%$.

The calorimeter[2] is divided into a barrel and two end-caps, and covers $98 \%$ of the solid angle. The modules are read-out at both ends by photo-multipliers, both in amplitude and time for a total of 2440 cells per side arranged in five layers. Cells close in time and space are grouped into calorimeter clusters. The cluster energy $E$ is the sum of the cell energies. The cluster time $T$ and position $\vec{R}$ are energy-weighed averages. Energy and time resolutions are $\sigma_{E} / E=5.7 \% / \sqrt{E(\mathrm{GeV})}$ and $\sigma_{t}=57 \mathrm{ps} / \sqrt{E(\mathrm{GeV})} \oplus 100 \mathrm{ps}$, respectively.

During KLOE data taking DA $\Phi$ NE beam conditions and detectors calibrations are constantly monitored in order to guarantee the highest quality of the collected data. Actually KLOE has already acquired $2.5 \mathrm{fb}^{-1}$ of data and a new extensive campaign of data taking is starting under the project KLOE-2[9].

The production cross section for the process $e^{+} e^{-} \rightarrow \phi$ is $3.3 \mu \mathrm{b}$ and the $\phi$ meson decays into $K_{0} \bar{K}_{0}$ with a branching fraction of $\sim 34 \%$. The initial state of the kaon pair is produced via strong interaction with quantum numbers $J^{P C}=1^{--}$:

$$
|i\rangle=\frac{\left|K_{0}\right\rangle\left|\bar{K}_{0}\right\rangle-\left|\bar{K}_{0}\right\rangle\left|K_{0}\right\rangle}{\sqrt{2}}=\mathscr{N}\left(\left|K_{S}(\vec{p})\right\rangle\left|K_{L}(-\vec{p})\right\rangle-\left|K_{S}(-\vec{p})\right\rangle\left|K_{L}(\vec{p})\right\rangle\right),
$$

where $\left|K_{S} / K_{L}\right\rangle$ are the kaon mass eigenstate and $\mathscr{N}$ is a normalization factor.

The eq. 1.1 implies that the detection of one of the two kaon on one side reveals the presence of the other kaon on the opposite side ${ }^{1}$. During KLOE data reconstruction this feature is used to select pure $K_{S}$ or $K_{L}$ beams identifying $K_{L}$ interaction in the EMC or $K_{S} \rightarrow \pi^{+} \pi^{-}$decay respectively ${ }^{2}$. The example of $K_{S}$ tagging using $K_{L}$ interaction in the EMC is shown in fig. 1-left.

A different approach can be exploited at KLOE since the two kaons are produced in an antisymmetric correlated state. Labeling $f_{1}$ and $f_{2}$ the decay channel for the two kaons, the time

\footnotetext{
${ }^{1}$ At KLOE, since the $\phi$ has a non zero momentum along $\mathrm{x}$, the two kaons are not perfectly back-to-back in the laboratory frame.

${ }^{2}$ In the second case a non overlapping fiducial volume has to be defined in order not to have misidentification of the kaon mass eigenstate.
} 
evolution of the initial state decaying in the given pair channels is expressed as a function of the difference of decay time $\left(\Delta t=t_{2}-t_{1}\right)$ as:

$$
I_{f_{1} f_{2}}(\Delta t) \propto e^{-\Gamma|\Delta t|}\left[\left|\eta_{1}\right|^{2} e^{\frac{\Delta \Gamma}{2} \Delta t}+\left|\eta_{2}\right|^{2} e^{\frac{\Delta \Gamma}{2} \Delta t}-2 \Re e\left(\eta_{1} \eta_{2} e^{-i \Delta m \Delta t}\right)\right]
$$

where $\eta_{j}=\left\langle f_{j} \mid K_{L}\right\rangle /\left\langle f_{j} \mid K_{S}\right\rangle, \Gamma=\Gamma_{S}+\Gamma_{L}$ and $\Delta \Gamma=\Gamma_{S}-\Gamma_{L}$. If the final states considered are the same for the two kaons $\left(f_{1}=f_{2}=f\right)$ it is not possible to distinguish between them. In the standard Quantum Mechanics description of the time evolution of the system, a fully destructive interference is expected for equal decay time $(|\Delta t|=0)$ as shown if fig. 1(right), where $f_{j}=\pi^{+} \pi^{-}$is assumed.
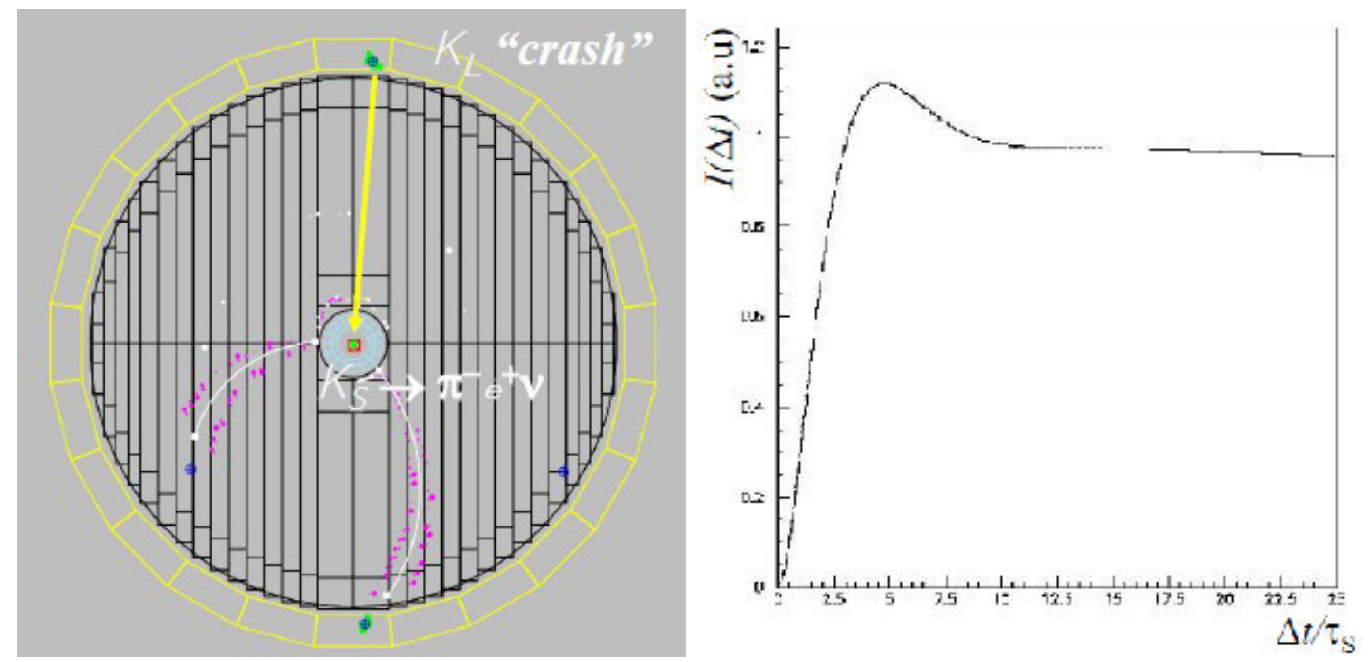

Figure 1: Description of the $K_{S}$ tagging (left). Distribution of eq. 1.2 in the assumption $f_{1}=f_{2}=\pi^{+} \pi^{-}$ and no final state or side identification (right).

\section{Measurement of $B R\left(K_{S} \rightarrow 3 \pi_{0}\right)$}

The decay of $K_{S}$ meson into three pions has not yet been observed, and the best published limit on the branching ratio $B R\left(K_{S} \rightarrow 3 \pi^{0}\right)<1.2 \times 10^{-7}$ [15] is about two orders of magnitude larger than predictions based on the Standard Model. Moreover, this process violates $C P$ symmetry and, assuming $C P T$ invariance, allows one to investigate direct $C P$ violation. At KLOE this decay is reconstructed by searching for events with a $K_{L}$ interaction in the calorimeter (so called $K_{L}$-crash), six photon clusters and no tracks from the interaction point. The background originates mainly from $K_{S} \rightarrow 2 \pi^{0}$ events with two spurious clusters from splittings or accidental activity or is due to false $K_{L}$-crash tags from $K_{S} \rightarrow \pi^{+} \pi^{-}$and $K_{L} \rightarrow \pi^{0} \pi^{0} \pi^{0}$ events. In the latter case, charged pions from $K_{S}$ decays interact in the low-beta insertion quadrupoles, ultimately simulating the $K_{L}$-crash signal, while $K_{L}$ decays close to the interaction point produce six real photons [15] matching the kaon hypothesis. To improve the reconstruction a global fit is performed. In the fitting procedure the kinematical constraints are used: energy and momentum conservation, the kaon mass and the velocities of the six photons.

The background events from false $K_{L}$-crash are considerably reduced by cutting on the $\chi^{2}$ of the global fit. In order to gain a good rejection of background from events with split or accidental 

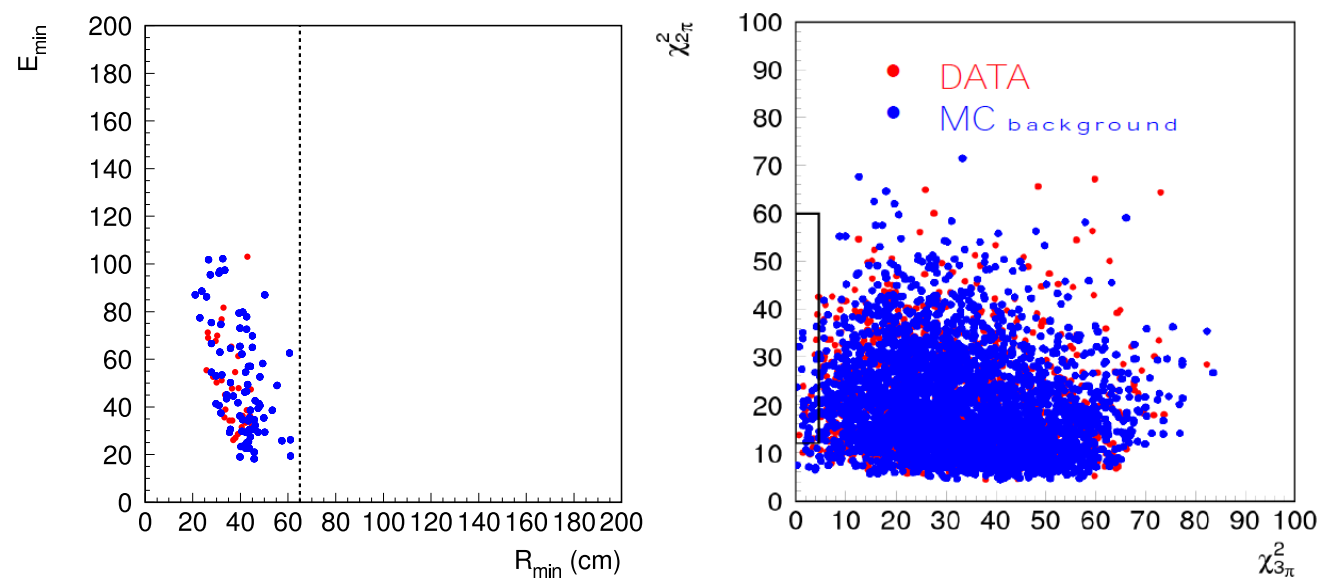

Figure 2: The distribution of the minimal energy of the cluster versus minimal distance $\left(R_{\min }\right)$ between clusters in the event for data (red) and MC (blue) (left). The dashed line corresponds to the $R_{\min }$ cut used. Distribution of $\chi_{3 \pi}^{2}$ vs $\chi_{2 \pi}^{2}$ for data (red) and MC (blue) (right). The signal box definition is shown. Both distributions are shown after the cut on the $\chi^{2}$ of the global fit.

clusters, the correlation between the following two $\chi^{2}$-like discriminating variables, $\chi_{3 \pi}^{2}$ and $\chi_{2 \pi}^{2}$, is used. $\chi_{3 \pi}^{2}$ is the quadratic sum of the residuals between the nominal $\pi^{0}$ mass and the invariant masses of three photon pairs formed from the six clusters present. $\chi_{2 \pi}^{2}$ is based instead on energy and momentum conservation in the $\phi \rightarrow K_{S} K_{L}, K_{S} \rightarrow \pi^{0} \pi^{0}$ decay hypothesis, as well as on the invariant masses of two photon pairs. Both variables are evaluated with the most favorable cluster pairing in each case [15]. In addition, in order to improve the quality of the photon selection using $\chi_{2 \pi}^{2}$, we cut on the variable $\Delta E=\left(m_{\Phi} c^{2} / 2-\sum E_{\gamma_{i}}\right) / \sigma_{E}$, where $\gamma_{i}$ is the $\mathrm{i}-t h$ photon from the four chosen in the $\chi_{2 \pi}^{2}$ estimator and $\sigma_{E}$ is the appropriate resolution. For $K_{S} \rightarrow 2 \pi^{0}$ decays plus two background clusters, we expect $\Delta E \sim 0$ while, for $K_{S} \rightarrow 3 \pi^{0}, \Delta E \sim m_{\pi^{0}} c^{2} / \sigma_{E}$. At the end of the analysis we cut also on the minimal distance between the photon clusters to refine the rejection of events with split clusters (see left panel of fig. 2).
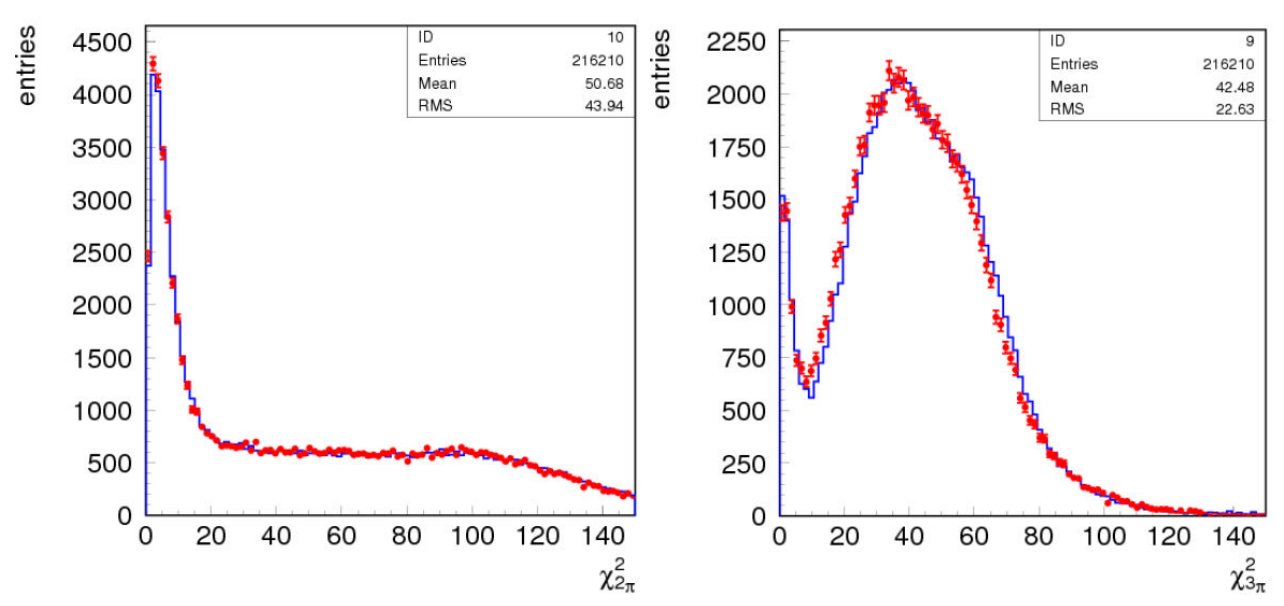

Figure 3: Distribution of $\chi_{2 \pi}^{2}$ (left) and $\chi_{3 \pi}^{2}$ (right). Red points are data and blue line are MC events. Both distributions are shown after the cut on the $\chi^{2}$ of the global fit and outside the signal box. 
The counting signal box is defined in the plane $\chi_{2 \pi}^{2}$ versus $\chi_{3 \pi}^{2}$. After each step of the selection the Data-MC agreement is checked in the region of the former plane out of the signal box. Very accurate MC calibrations has been performed in order to reach the high level of agreement shown in fig. 3 .

Applying the preliminary selection cuts, we find zero candidates in $1.7 \mathrm{fb}^{-1}$ of real data with zero background events expected from Monte Carlo, corresponding to an effective statistics of two times that of the data. This results in a new preliminary upper limit on the branching ratio:

$$
B R\left(K_{S} \rightarrow 3 \pi^{0}\right)<2.9 \times 10^{-8} @ 90 \% \text { C.L. }
$$

\section{3. $C P T$ and Lorentz symmetry breaking}

The $C P T$ breaking could be observed using kaon interferometry with both kaons decaying in $\pi^{+} \pi^{-}$pairs. In this case the only way to label the two final state is to use their momenta to define two "sides" like in the tagging. The eq. 1.2 becomes:

$$
I_{ \pm}(\Delta t) \propto e^{-\Gamma|\Delta t|}\left[\left|\eta_{ \pm}\left(\vec{p}_{1}\right)\right|^{2} e^{\frac{\Delta \Gamma}{2} \Delta t}+\left|\eta_{ \pm}\left(\vec{p}_{2}\right)\right|^{2} e^{\frac{\Delta \Gamma}{2} \Delta t}-2 \Re e\left(\eta_{ \pm}\left(\vec{p}_{1}\right) \eta_{ \pm}^{*}\left(\vec{p}_{2}\right) e^{-i \Delta m \Delta t}\right)\right]
$$

Allowing for $C P T$ violation $\eta_{ \pm}=\varepsilon_{K}-\delta_{K}$, where $\varepsilon_{K}$ is the $C P$ violation parameter in the Kaon system, while $\delta_{K}$ represents the $C P T$ violation.

A general theoretical possibility for $C P T$ violation is based on spontaneous breaking of Lorentz symmetry, as developed by Kostelecky[3, 4, 5]: the Standard-Model Extension (SME). Following this theoretical approach, for neutral kaons, $C P T$ violation manifests to lowest order only in the parameter $\delta_{K}$ and is related to Lorentz Invariance breaking. This results in a dependence of $\delta_{K}$ on the 4-momentum of the kaon:

$$
\delta_{K} \approx i \sin \phi_{S W} e^{i \phi_{S W}} \gamma_{K}\left(\Delta a_{0}-\vec{\beta}_{K} \cdot \Delta \vec{a}\right) / \Delta m
$$

where $\gamma_{K}$ and $\vec{\beta}_{K}$ are the kaon boost factor and velocity in the observer frame, and $\Delta a_{\mu}$ are the $C P T$ violation coefficients for the two valence quarks in the kaon.

The rotation of the Earth will results in a dependence of the $\delta_{K}$ parameter to the sidereal time. Choosing a three-dimensional basis $(\hat{X}, \hat{Y}, \hat{Z})$ in a non-rotating frame, with the $\hat{Z}$ axis along the Earth's rotation axis, and a basis $(\hat{x}, \hat{y}, \hat{z})$ for the laboratory frame[4] (see fig. 4 ) the eq. 3.2 will transform as:

$$
\begin{aligned}
\delta_{K}\left(\vec{p}_{K}, t\right)=\frac{i \sin \phi_{S W} e^{i \phi_{S W}}}{\Delta m} \gamma_{K}[ & \Delta a_{0}+\beta_{K} \Delta a_{Z}(\cos \vartheta \cos \chi-\sin \vartheta \cos \phi \sin \chi) \\
& -\beta_{K} \Delta a_{X} \sin \vartheta \sin \phi \sin \Omega T \\
& +\beta_{K} \Delta a_{X}(\cos \vartheta \sin \chi+\sin \vartheta \cos \phi \sin \chi) \cos \Omega T \\
& +\beta_{K} \Delta a_{Y}(\cos \vartheta \sin \chi+\sin \vartheta \cos \phi \sin \chi) \sin \Omega T \\
& \left.-\beta_{K} \Delta a_{Y} \sin \vartheta \sin \phi \cos \Omega T\right],
\end{aligned}
$$

where $T$ is the sidereal time, $\Omega$ is the Earth's sidereal frequency and $\chi$ is the angle between the Earth rotation axis and the $\hat{z}$ direction in the laboratory frame. 

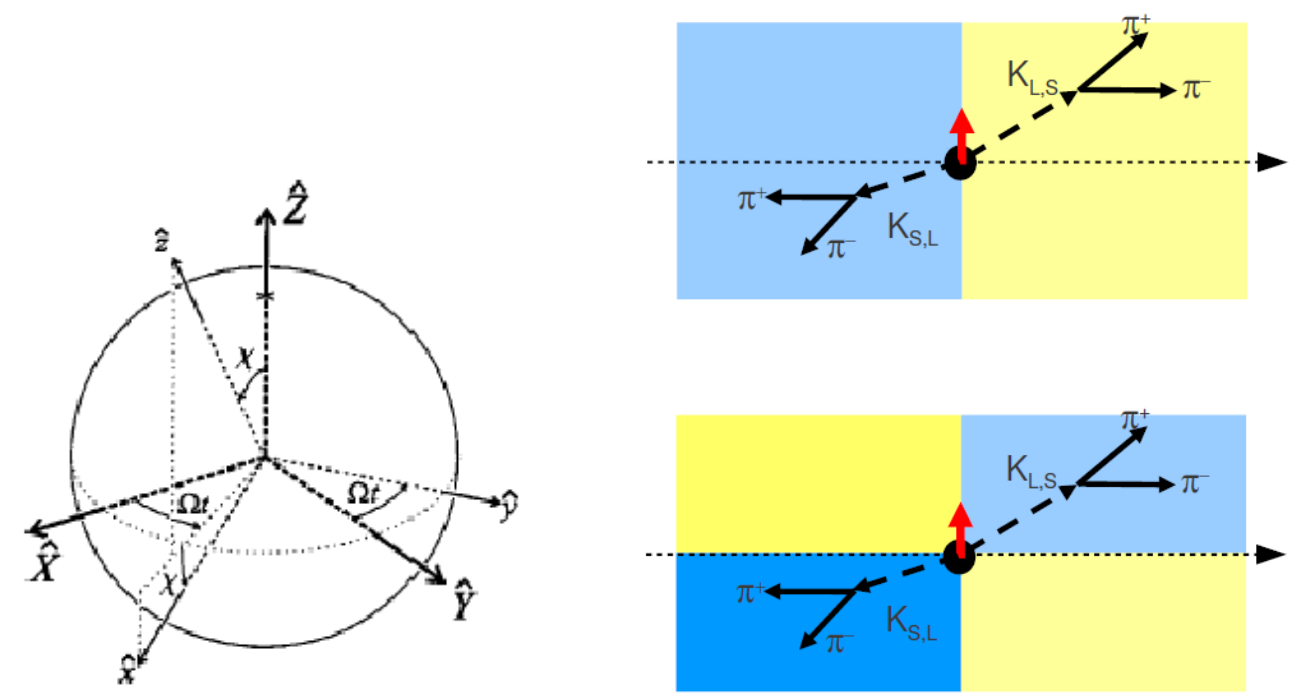

Figure 4: Description of the sidereal reference frame and the relative coordinate transformation (left). Top view of the KLOE orizontal cross section. The two plots shown the different sampling criteria used in the preliminary analysis (top) and current approach (bottom). For the preliminary measurements kaons are ordered according to their momenta along $\mathrm{z}$ axis and distribution of eq. 3.1 is observed as a function of sidereal time only. In the new method descripted, two angular bins are considered (I-III and II-IV quadrant in the picture). This will allow to observe asymmetry due to $\Delta a_{0}$ parameter that will be integrated to zero in the previous apporach. The red arrow represents the $\phi$ momentum.

Every $\Delta a_{\mu}$ components in the eq. 3.3 has his own dependence with respect to sidereal time and kaon flight direction. This kind of dependence will be exploited in our analysis of the KLOE data to access the complete set of SME parameters.

In the KLOE experiment the two kaons decay with opposite momenta ${ }^{3}$ thus experiencing, on a event by event basis, two different modulation for the $\delta_{K}$ parameter appearing in $\eta_{ \pm}$coefficients as described in eq. 3.2. Because of eq. 3.3 we expect small asymmetries in the two branches of the function $(\Delta t<0$ vs $\Delta t>0)$ that should exhibit the expected modulation as a function of sidereal time and kaon directions. The selection chain is fully symmetric for the two decay vertex in order to minimize any possible systematic effect induced by the analysis criteria. The background contamination in the sample is of the order of $1.7 \%$ and is manly due to the kaon regeneration on the beam pipe. The signal has a very clean topology, two decay vertex reconstructed from two pairs of tracks, we exploit the possibility to use global event information in the signal hypothesis to improve the reconstruction as shown in fig. 5-left.

In order to access all the $\Delta a_{\mu}$ parameters the data collected has to be analyzed as a function of their sidereal time and kaon direction. As shown in fig. 4-bottom right the data sample is divided in two subset for which the kaons going in the forward direction has higher (lower) value for $\gamma_{K}$ since it is in emitted along (opposite) to the $\phi$ boost. In this way it is possible to observe the asymmetry induced by the eq. 3.3 in the eq. 3.1 because of the different kaons momenta: directions

\footnotetext{
${ }^{3}$ Two kaons are not produced exactly back to back because of the $\phi$ boost in the lab frame. This small effect will be fundamental to access all the four $\Delta a_{\mu}$ parameters as discussed in the text.
} 

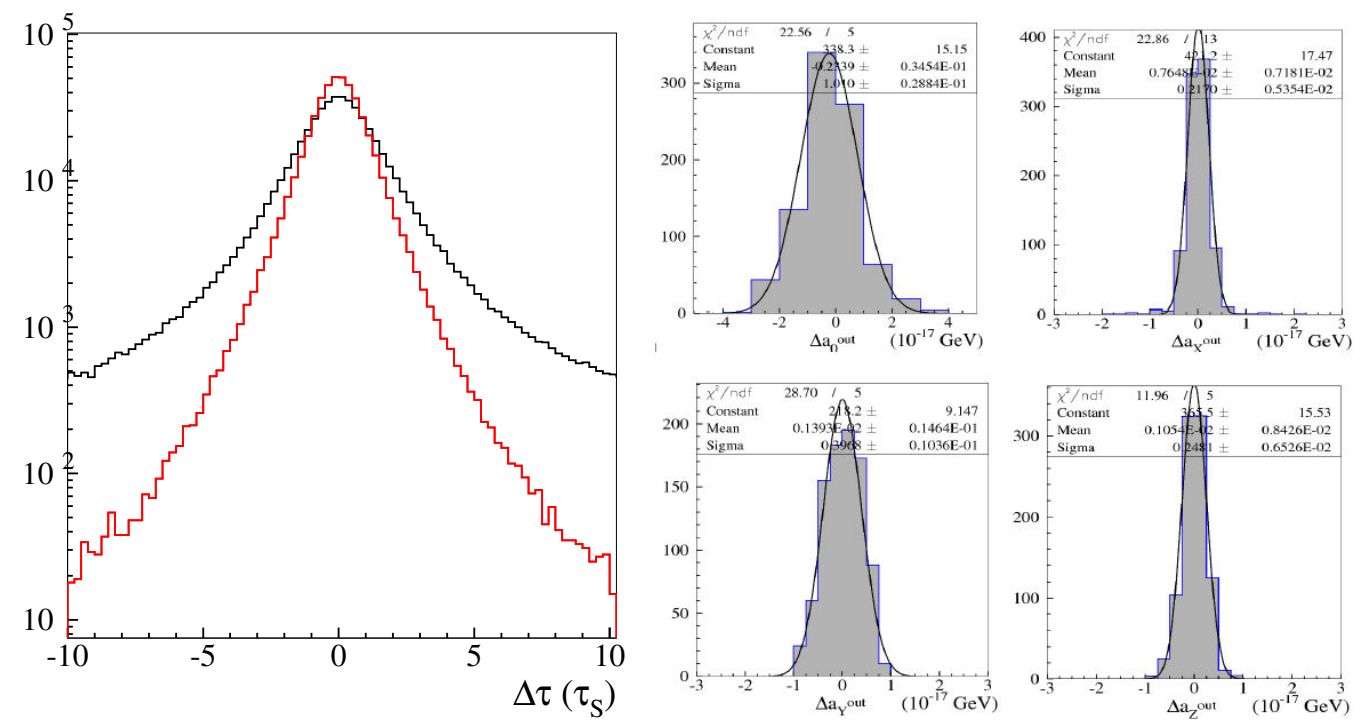

Figure 5: Resolution on the proper decay time difference (left). The black is the output of the standard reconstruction, the red one is the output of the global fit. Only the events passing the global fit are shown.

and modules. The expected sensitivity with this method, as show in fig. 5, is $10^{-17} \mathrm{GeV}$ for $\Delta a_{0}$ and ranging between $2-5 \times 10^{-18}$ for $\Delta \vec{a}$.

With KLOE data we already performed a preliminary analysis [8] using only half of the total available statistics $\left(1 \mathrm{fb}^{-1}\right)$. In this analysis we use the forward-backward asymmetry along $\mathrm{z}$ direction as a function of sidereal time only (e.g. integrating over azimuthal angle see fig. 4-topright). With this approach only the vector part of the $\Delta a_{\mu}$ four-vector can be observed ${ }^{4}$. Our preliminary results on $\Delta a_{X, Y, Z}$ are:

$$
\begin{array}{r}
\Delta a_{X}=(-6.3 \pm 6.0) \times 10^{-18} \mathrm{GeV}, \\
\Delta a_{Y}=(2.8 \pm 5.9) \times 10^{-18} \mathrm{GeV}, \\
\Delta a_{Z}=(2.4 \pm 9.7) \times 10^{-18} \mathrm{GeV} .
\end{array}
$$

\section{Conclusions and future plans}

The continuation of the KLOE physics program with KLOE-2 [9] at an improved DAФNE machine is currently starting with a new beam interaction scheme [10] and with the inclusion of two pairs of electron-positron taggers [11] for the study of the gamma-gamma physics.

Several other upgrades for the detector are foreseen in the next future:

- a pair of crystal calorimeters (CCALT[12]) near the interaction region to improve the angular acceptance for low- $\theta$ particles;

\footnotetext{
${ }^{4}$ The integration over azimuthal angle does not allow to observe as a function of kaon momenta value (e.g. $\gamma_{K}$ ). According to eq. 3.3 this will wash out the $\Delta a_{0}$ contribution to the asymmetry.
} 
- a pair of tile calorimeters (QCALT[13]) covering the quadrupoles along the beam pipe made of tungsten foil and singly read-out scintillator tiles to improve the angular coverage for particles coming from the active volume of the DC;

- a small and light tracker (IT[14]) made of four planes of cylindrical GEM to improve the resolution of the vertex reconstruction around the interaction point and to increase the low- $\theta$ charged particles acceptance.

The results presented are expected to be improved with the KLOE-2 data taking campaign. The $K_{S} \rightarrow 3 \pi^{0}$ will benefit either by the increased statistics either by the presence of the low $\theta$ calorimeters (CCAL-T and QCAL-T) that will increase the selection efficiency and background vetoing. With the expected KLOE-2 dataset a first observation of such decay should become possible. The sensitivity of $C P T \&$ Lorentz invariance tests will improve either by the increased statistics either by the IT presence and interaction scheme. The former will improve the resolution on the vertex position and the acceptance for low- $\theta$ tracks, the latter will implies a larger beam crossing angle (from 25 to $60 \mathrm{mrad}$ ) that will enhance the effect of asymmetry between the two kaons. The expected sensitivity should increase up to $10^{-19} \mathrm{GeV}$ for all the $\Delta a_{\mu}$ parameters.

\section{References}

[1] M. Adinolfi et al., KLOE Collaboration, Nucl. Inst. Meth. A 488 (2002) 51.

[2] M. Adinolfi et al., KLOE Collaboration Nucl. Inst. Meth. A 482 (2002) 363.

[3] V.A. Kostelecký, Phys. Rev. Lett. 80, 1818 (1998).

[4] V.A. Kostelecký, Phys. Rev. D 61, 016002 (1999).

[5] V.A. Kostelecký, Phys. Rev. D 64, 076001 (2001).

[6] A. Di Domenico, in V.A. Kostelecký, ed., Fourth Meeting on CPT and Lorentz Symmetry, World Scientific, Singapore, 2008; A. Di Domenico, in A. Di Domenico ed., Handbook on neutral kaon interferometry at a $\Phi$-factory, Frascati Physics Series, Vol. 43.

[7] C. D. Buchanan et al., Phys. Rev. D 45, 4088 (1992).

[8] A. Di Domenico, J. Phys. Conf. Ser. 171, 012008 (2009).

[9] G. Amelino-Camelia et al., arXiv:1003.3868.

[10] C. Milardi et al., DAFNE Collaboration, arXiv:1006.1487.

[11] D. Babusci et al., Nucl. Instrum. Meth. A 617, 81 (2010).

[12] F. Happacher et al., Nucl. Phys. Proc. Suppl. 197, 215 (2009).

[13] M. Cordelli et al., Nucl. Instrum. Meth. A 617, 105 (2010).

[14] F. Archilli et al., KLOE-2 Collaboration, arXiv:1002.2572.

[15] F. Ambrosino, et al., Phys. Lett. B 619, 61-70 (2005). 